\title{
Retraction Note to: Efficacy of favipiravir in COVID-19 treatment: a multi-center randomized study
}

\author{
Hany M. Dabbous ${ }^{1} \cdot$ Sherief Abd-Elsalam ${ }^{2}$ (D) - Manal H. El-Sayed ${ }^{3}$. Ahmed F. Sherief ${ }^{1} \cdot$ Fatma F. S. Ebeid $^{3}$. \\ Mohamed Samir Abd El Ghafar ${ }^{4}$. Shaimaa Soliman ${ }^{5}$. Mohamed Elbahnasawy ${ }^{6} \cdot$ Rehab Badawi $^{2}$. \\ Mohamed Awad Tageldin ${ }^{7}$
}

Published online: 22 November 2021

○) Springer-Verlag GmbH Austria, part of Springer Nature 2021

\section{Retraction to: Archives of Virology (2021) 166:949-954 https://doi.org/10.1007/s00705-021-04956-9}

The Editor-in-Chief has retracted this article. After publication, concerns were raised about the reporting of this clinical trial and the authors were asked to provide their raw data files. The raw data underlying Table 1 were examined. First, the reported baseline variables showed that the distribution of one variable was highly statistically different in the two study groups. Second, two variables showed different rounding to significant figures in the two groups. Third, for two variables, there was a different distribution of the variables when moving through the groups. It is unclear how these variations could occur in a

The original article can be found online at https://doi.org/10.1007/ s00705-021-04956-9.

Sherief Abd-Elsalam

sherif.abdelbaky@med.tanta.edu.ed

Hany M. Dabbous

drhdabbous@gmail.com

Manal H. El-Sayed

manalhelsayed@yahoo.co.uk

Ahmed F. Sherief

Ahmedfouad85@live.com

Fatma F. S. Ebeid

dr.fatma_ebeid@yahoo.com

Mohamed Samir Abd El Ghafar

mohamed.abdelghafar1@med.tanta.edu.eg

Shaimaa Soliman

shaimaasherif@hotmail.com

Mohamed Elbahnasawy

drmogabehn@gmail.com

Rehab Badawi

dr.rb.badawi@gmail.com

Mohamed Awad Tageldin

mohamedawadtag@yahoo.com correctly performed trial so that serious doubts must arise on the randomization process and on the data validity. These doubts are reinforced by the equal gender distribution even though gender was not stated to be an inclusion parameter by the authors. The Editor-in-Chief therefore no longer has confidence in the results and conclusions presented. Sherief Abd Elsalam and Shaimaa Soliman disagree with this retraction. Hany M. Dabbous, Manal H. El Sayed, Ahmed F. Sherief, Fatma F. S. Ebeid, Mohamed Samir Abd El Ghafar, Mohamed Elbahnasawy, Rehab Badawi and Mohamed Awad Tageldin have not responded to correspondence from the publisher about this retraction.

Publisher's Note Springer Nature remains neutral with regard to jurisdictional claims in published maps and institutional affiliations.

1 Tropical Medicine Department, Faculty of Medicine, Ain Shams University, Cairo, Egypt

2 Department of Tropical Medicine and Infectious Diseases, Faculty of Medicine, Tanta University, El-Giash Street, Tanta 31527, Egypt

3 Faculty of Medicine, Ain Shams University Research Institute-Clinical Research Center (MASRI-CRC), Cairo, Egypt

4 Department of Anesthesia, Surgical Intensive Care and Pain Medicine, Faculty of Medicine, Tanta University, Tanta, Egypt

5 Public Health and Community Medicine, Menoufia University, Menoufia, Egypt

6 Emergency Medicine and Traumatology Department, Faculty of Medicine, Tanta University, Tanta, Egypt

7 Department of Chest Diseases, Faculty of Medicine, Ain Shams University, Cairo, Egypt 